\title{
Six Years of Bursts with the SPI-ACS
}

\author{
Kevin Hurley ${ }^{1}$ \\ University of California Space Sciences Laboratory \\ 7 Gauss Way, Berkeley, CA, 94720-7450 U.S.A. \\ E-mail: khurley@ssl.berkeley.edu
}

\section{Arne Rau}

California Institute of Technology

1200 California Blvd., MS 105-24, Pasadena, CA, 91125 U.S.A.

E-mail: arne@astro.caltech.edu

\section{Giselher Lichti}

Max-Planck-Institut für extraterrestrische Physik

Giessenbachstrasse Postfach 1312, Garching, 85748 Germany

E-mail: grl@mpe.mpg.de

\section{Andreas von Kienlin}

Max-Planck-Institut für extraterrestrische Physik

Giessenbachstrasse Postfach 1312, Garching, 85748 Germany

E-mail: azk@mpe.mpg.de

The anticoincidence system of the INTEGRAL spectrometer has been an essential component of the interplanetary network since launch. It has observed about 600 events which have been confirmed as either soft gamma repeaters or cosmic gamma ray bursts by other instruments in the IPN. It has also observed over 130 events which are unconfirmed, but which are almost certainly weak bursts below the thresholds of the other IPN experiments. We review the highlights of these observations, which include gamma-ray bursts, soft gamma repeaters, and one or two extragalactic giant magnetar flares.

The 7th INTEGRAL Workshop

Copenhagen, Denmark

September 8- 112008

\footnotetext{
$1 \quad$ Speaker
} 


\section{Introduction}

The SPI anticoincidence system was first proposed as a gamma-ray burst (GRB) detector at the 2nd INTEGRAL workshop in St. Malo in 1996 (Hurley 1997). Its BGO shield, which surrounds the spectrometer on the bottom and sides, has a maximum effective area of up to $5250 \mathrm{~cm}^{2}$ at $100 \mathrm{keV}$, and a thickness of 1.6 to $5 \mathrm{~cm}$. For comparison, a BATSE module had $4000 \mathrm{~cm}^{2}$, the Swift BAT has $5240 \mathrm{~cm}^{2}$, and the Fermi Burst Monitor has $200 \mathrm{~cm}^{2}$. The SPIACS has a superior response above $100 \mathrm{keV}$. Its effective area is a function of azimuthal and zenithal angles, and drops to about $500 \mathrm{~cm}^{2}$ on-axis. When triggered, it produces $105 \mathrm{~s}$ long GRB time histories with $50 \mathrm{~ms}$ resolution in a single energy channel which ranges from $\sim 100$ $\mathrm{keV}$ to $10 \mathrm{MeV}$. It does not record energy spectra, nor does it have an independent GRB localization capability.

\section{The Score So Far}

The SPI-ACS has now detected about 550 confirmed cosmic gamma-ray bursts (1 every 3 days); these are events which have been observed by at least one other experiment in the interplanetary network (IPN). The weakest confirmed event is GRB071112, with a fluence of $4.8 \times 10^{-8} \mathrm{erg}^{-2}$. Its time history is shown in figure 1 . The total trigger rate is extremely variable, ranging from one to several hundred triggers per day. Some of these are random fluctuations, while others are due to noise or particles. Others, however, are almost certainly weak gamma-ray bursts which are unconfirmed because they are below the thresholds of the other IPN experiments; 135 have been detected so far. The SPI ACS has detected about 45 short bursts and one giant flare from the magnetar SGR1806-20 (Mereghetti et al. 2005), and one short burst from SGR1900+14. No events have been detected from the 'new' magnetar SGR0501+4516, probably because the bursts have been relatively weak.

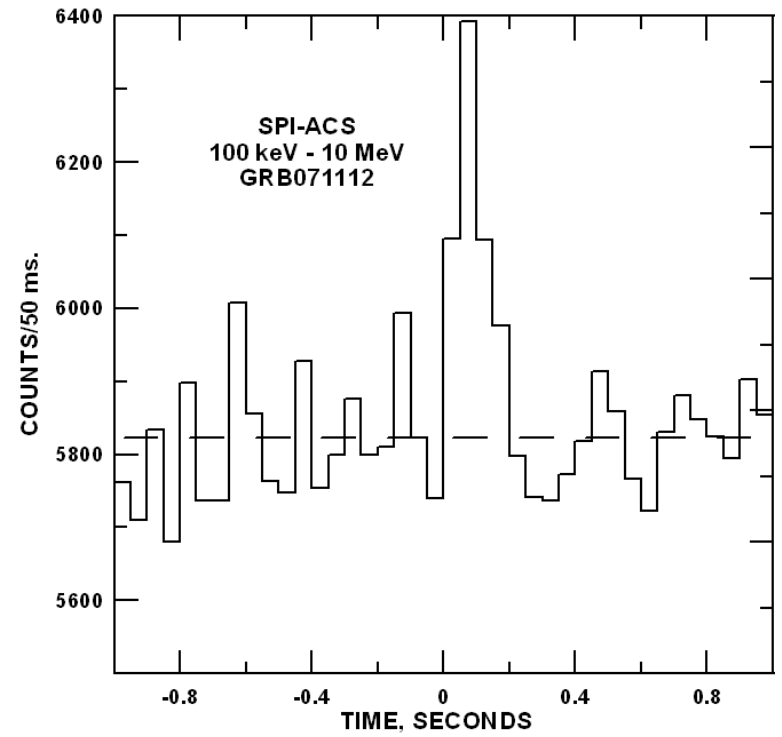

Figure 1. The weakest confirmed cosmic event observed by SPI-ACS. This burst, which resembles many of the weak unconfirmed events, was also detected by Swift. 


\section{Science With The SPI-ACS Data}

The science which can be done with the SPI-ACS data falls into three broad categories:

1. detailed light curve studies which take advantage of the excellent statistics and high energy sensitivity of the ACS,

2. Obtaining precise burst localizations with the IPN, and searching for electromagnetic counterparts, and

3. Searching for gravitational radiation, neutrino emission, and very high gamma-ray emission from bursts, more or less independently of the rapidity and accuracy of the localizations.

We discuss each of these in turn.

\subsection{Light Curve Studies}

Figure 2 shows the light curve of GRB021206. With a fluence of $1.6 \times 10^{-4} \mathrm{erg} \mathrm{cm}^{-2}$, this is one of the most intense events observed by the ACS. The statistics are excellent: up to 60,000 counts are recorded in a single $50 \mathrm{~ms}$ time bin. A gamma-ray afterglow is evident in this light curve; these are only detected in the strongest bursts, when they are observed with excellent statistics.

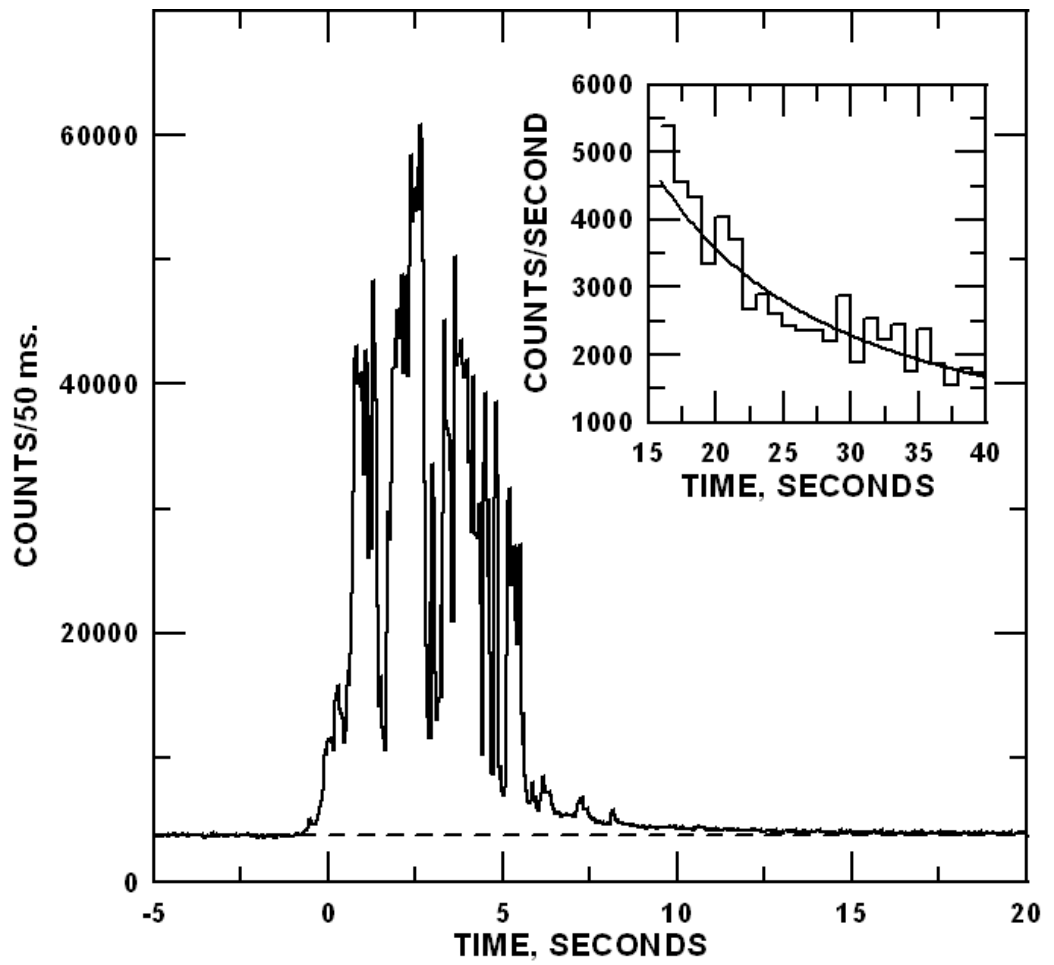

Figure 2. The main figure shows the light curve of GRB021206, with the background indicated by a dashed line. The inset shows the later part of the light curve after background subtraction. A gamma-ray afterglow is evident, which can be fit with a power law of index $\sim-1.1$. 
Gamma-ray afterglows following intense GRBs have been observed by other instruments prior to GRB021206. However, the observation by the SPI-ACS of a gamma-ray afterglow following the giant flare from SGR1806-20 was completely unexpected. This phenomenon, which was confirmed by RHESSI and Konus, lasted about one hour, and its spectrum extended to at least $1 \mathrm{MeV}$. The light curve is shown in figure 3 .

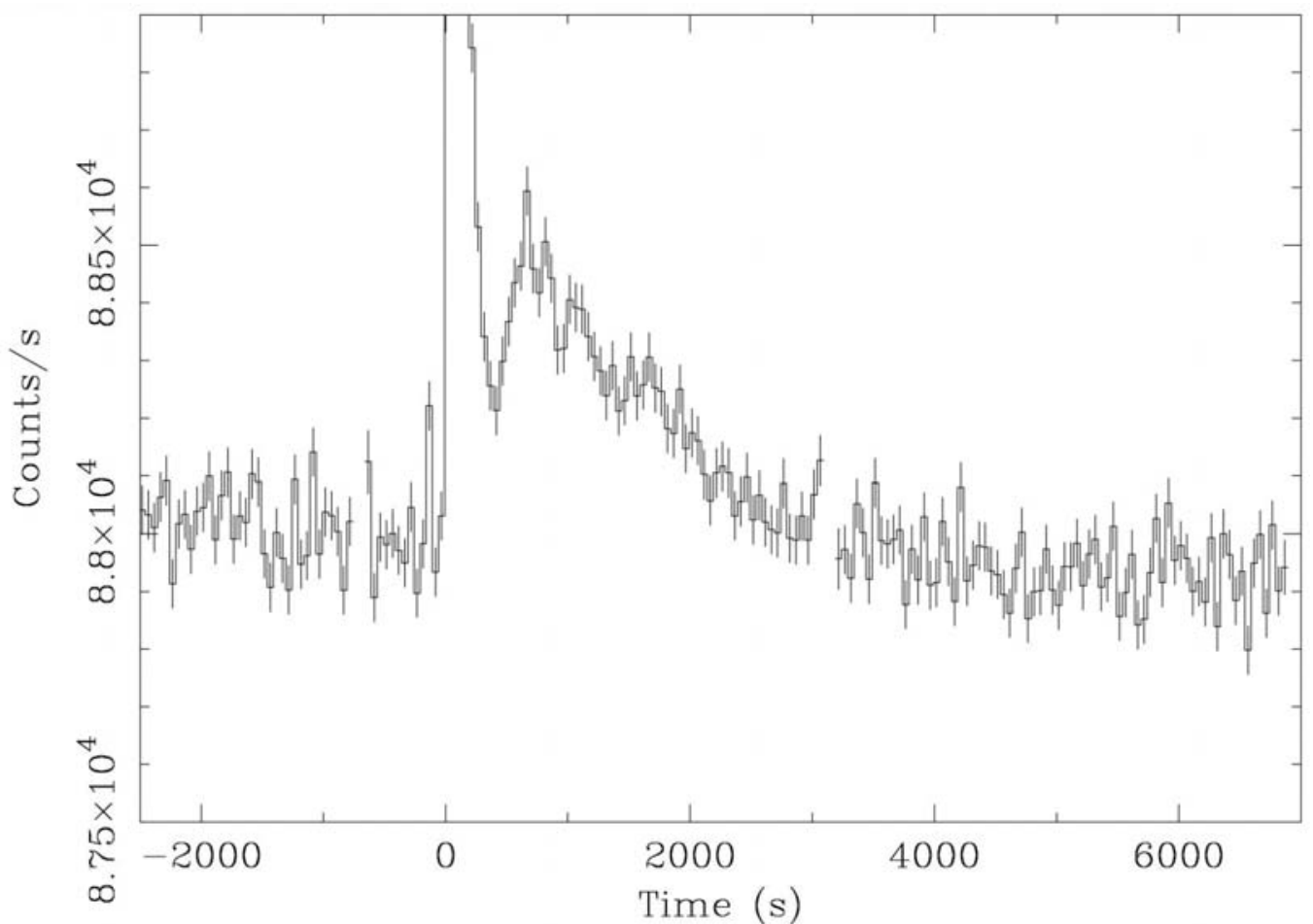

Figure 3. From Mereghetti et al. 2005. The SPI-ACS light curve of the SGR1806-20 giant flare with $50 \mathrm{~s}$ binning. The flare itself is evident starting around 0 seconds. The afterglow starts around the end of the flare, peaks around $500 \mathrm{~s}$, and continues for about one hour. Reproduced by permission of the AAS. DOI: 10.1086/430669

Since radio data on giant flares provide evidence for an expanding outflow from the magnetar, the afterglow may be related to it.

\subsection{SPI-ACS in the $3^{\text {rd }}$ Interplanetary Network.}

The SPI-ACS is one of nine instruments in the IPN. The others are RHESSI, the Suzaku WAM, AGILE (Super-AGILE and the mini-calorimeter), and the Swift BAT, all in low Earth orbit. (The Fermi GBM is being added to the network currently.) Konus-Wind is at about 5 light-seconds from Earth. Finally, MESSENGER, on its way to Mercury, and Mars Odyssey, in orbit around Mars, provide the interplanetary baselines. The configuration of the network is shown in figure 4. 


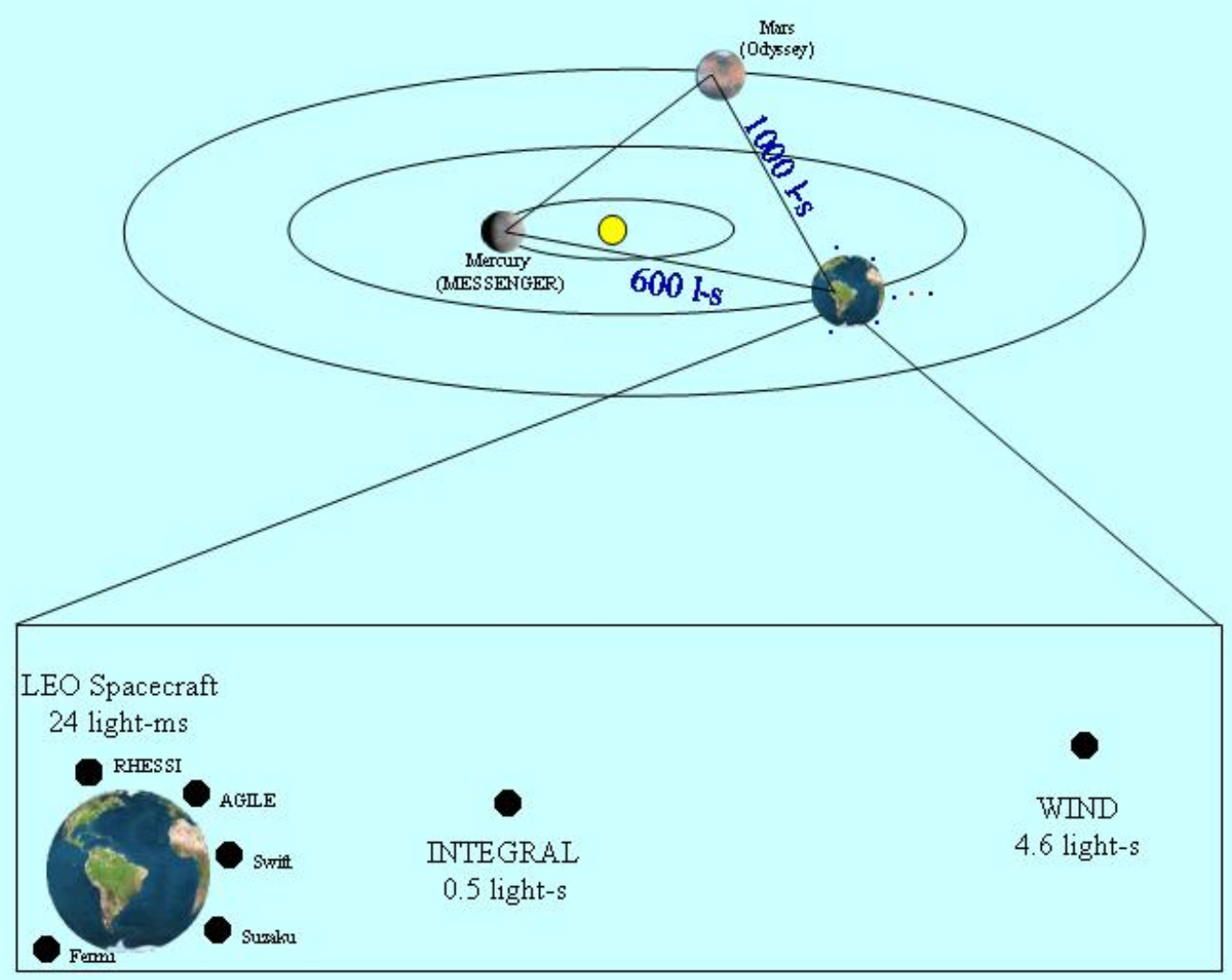

Figure 4. The present configuration of the Interplanetary Network.

The INTEGRAL spacecraft occupies a unique position in the network: it is far enough from Earth to provide a statistically independent vertex for GRB localization. About 200 bursts per year are detected by the IPN, and in general, they are not the same ones that narrow-field instruments such as IBIS and the Swift BAT observe. They are the more intense events, with fluences greater than $10^{-6} \mathrm{erg} \mathrm{cm}^{-2}$, and/or peak fluxes above 0.4 photon $\mathrm{cm}^{-2}$. Because INTEGRAL, Wind, and MESSENGER have fields of view that are unocculted by planetary bodies, the IPN constitutes an all-sky monitor, and it is in continuous operation, when the duty cycles of all its instruments are considered.

Two interesting events which were detected by the ACS are bursts on November 32005 and February 1 2007. Their light curves are shown in figure 5. 

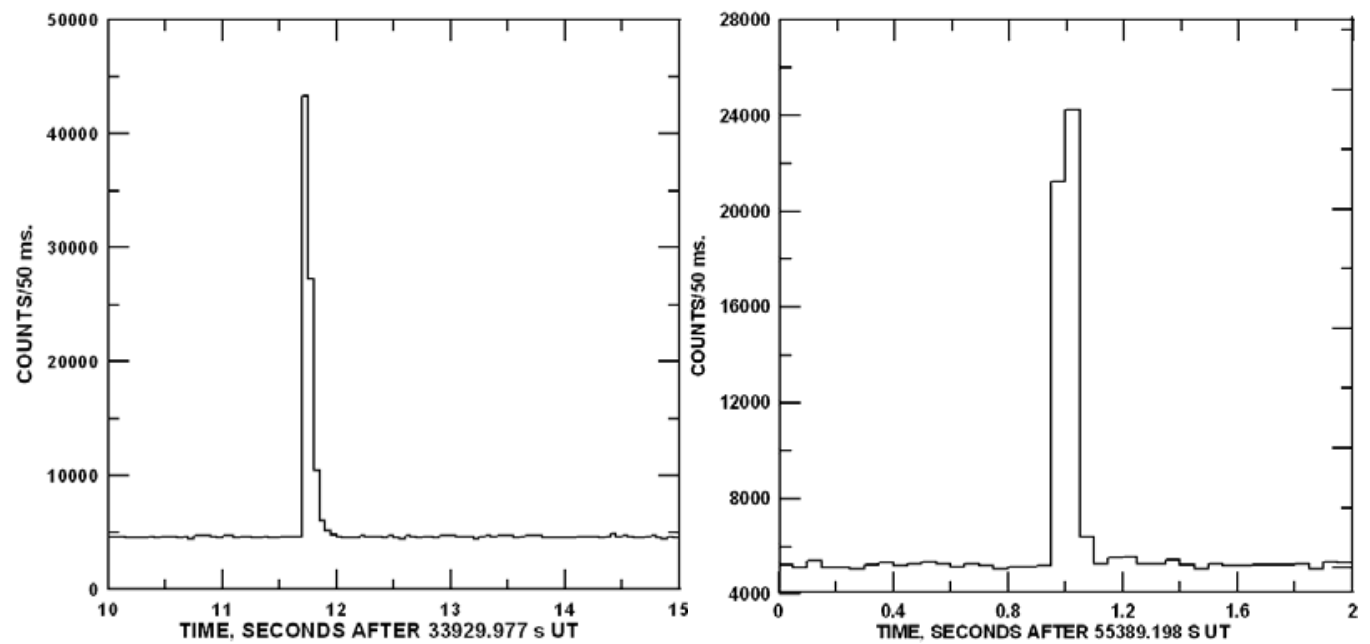

Figure 5. The SPI-ACS light curves of the November 32005 (left) and February 12007 (right) events.

The IPN localizations of these events are shown in figure 6. Because of their overlaps with bright galaxies, as well as their light curves and energy spectra, these bursts are quite likely to be extragalactic giant magnetar flares from M81 and M31 respectively (Frederiks et al. 2007, Mazets et al. 2008). If so, their isotropic gamma-ray energies were $7 \times 10^{46}$ and $1.5 \times 10^{45} \mathrm{erg}$, respectively.

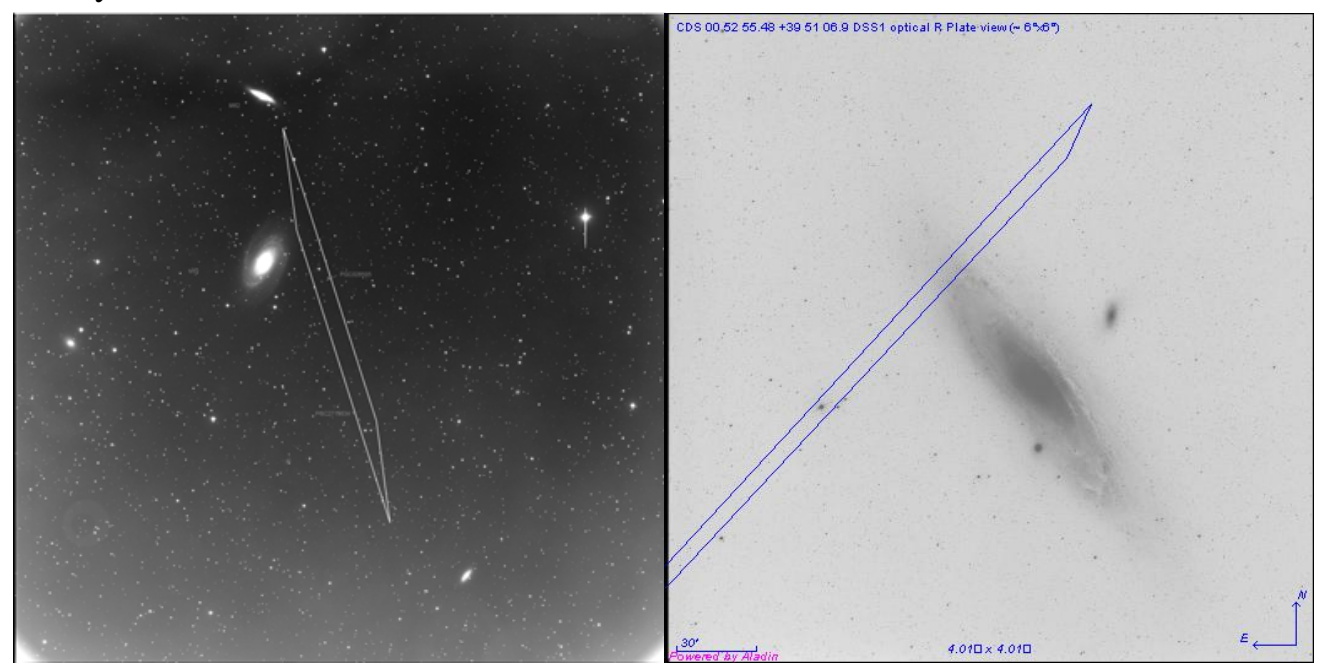

Figure 6. IPN error boxes for the November 32005 (left) and February 12007 (right) bursts. The outer contours of M81 are not visible in the left figure, but they overlap the IPN error box.

\subsection{Searches for Exotic Counterparts}

All-sky experiments, and experiments which view a large fraction of the sky, and record their data, often do not require fast or precise GRB localizations to determine whether an event can be correlated with a GRB. The trigger time alone is often sufficient to search for more exotic radiation, such as gravitational and neutrino radiation, and very high energy gamma-ray 
emission. LIGO, AMANDA, Pi of the Sky, and Milagro are in this category. These experiments benefit from having a large number of bright (and presumably nearby), isotropically distributed GRBs to study. Some results of these studies have appeared in Abbott et al. (2008), Achterberg et al. (2008), and Abdo et al. (2007). So far, they are all upper limits, but the Abbott et al. LIGO result is particularly interesting, since it demonstrates that the 070201 event could not have been caused by a binary merger in M31.

\section{Summary}

The INTEGRAL SPI-ACS is a sensitive monitor of fast gamma-ray transients in the fluence range $4.8 \times 10^{-8} \mathrm{erg} \mathrm{cm}^{-2}$ and above. Using these data, over 175 GCN Circulars have been issued, and GCN Notices have been issued starting around October 2008. About 90 of the bursts detected by the ACS have radio, optical, and/or X-ray counterparts, and for 38 events, a spectroscopic or photometric redshift has been determined; the redshifts lie in the range 0.105 to 3.35. Both the ACS light curve data, as well as the IPN data, are made publicly available at ftp://isdcarc.unige.ch/arc/FTP/ibas/spiacs/ and ssl.berkeley.edu/ipn3/index.html, respectively.

Acknowledgment. KH is grateful for support under the U.S. INTEGRAL Guest Observer program, NASA grant NNX08AC90G.

\section{References}

[1] K. Hurley, INTEGRAL as a Gamma-Ray Burst Detector in the Fourth Interplanetary Network, in The Transparent Universe, Proc. 2nd INTEGRAL Workshop, Eds. C. Winkler, T. Courvoisier, and Ph. Durouchoux, ESA SP 382, p. 491, 1997

[2] S. Mereghetti, D. Gotz, A. von Kienlin, A. Rau, G. Lichti, G. Weidenspointner, G., and P. Jean, The First Giant Flare from SGR1806-20: Observations Using the Anticoincidence Shield of the Spectrometer on INTEGRAL, Ap. J. 624, L105, 2005

[3] D. Frederiks, V. Palshin, R. Aptekar, S. Golenetskii, T. Cline, and E. Mazets, On the Possibility of Identifying the Short Hard Burst GRB051103 with a Giant Flare from a Soft Gamma Repeater in the M81 Group of Galaxies, Astron. Lett. 33(1), 19, 2007

[4] E. Mazets, R. Aptekar, T. Cline, D. Frederiks, J. Goldsten, S. Golenetskii, K. Hurley, A. von Kienlin, and V. Palshin, A Giant Flare from a Soft Gamma Repeater in the Andromeda Galaxy (M31), Ap. J. 680, 545, 2008

[5] A., Achterberg et al., The Search for Muon Neutrinos from Northern Hemisphere Gamma-Ray Bursts with AMANDA, Ap. J. 674, 357, 2008

[6] B. Abbott et al., Implications for the Origin of GRB 070201 from LIGO Observations, Ap. J. 681, 1419, 2008

[7] A. Abdo et al., Milagro Constraints on Very High Energy Emission from Short-Duration GammaRay Bursts, Ap. J. 666, 361, 2007 